\title{
Cómo realizar una evaluación de deglución completa, eficaz y en corto tiempo
}

\author{
How to make a complete, efficient and short-time evaluation of deglution
}

\author{
Arteaga J Patricia1 ${ }^{1}$, Olavarría L Christian ${ }^{1}$, Naranjo D Benjamín², Elgueta L Francisca², \\ Espínola M Daniella².
}

\section{RESUMEN}

日 trastorno de deglución es una patología que va en aumento en los pacientes hospitalizados. 1 estudio y manejo de esta condición tiene muchas alternativas según la bibliografía Analizamos las interconsultas para evaluación de deglución enviadas a nuestro servicio entre el 1 de enero al 31 de diciembre de 2004. Se confeccionó un protocolo con los antecedentes y el examen realizado y se clasificó a los pacientes según la severidad del trastorno observado ingresando así a un algoritmo diseñado para continuar su terapia y seguimiento.

Este trabajo pudo comprobar la alta incidencia del trastorno de deglución en pacientes hospitalizados; que correspondió al $20 \%$ del total de interconsultas del Servicio. Las dos causas más frecuentes para este trastorno fueron: accidente vascular en un 56\% e intubación prolongada en un 58\%; algunos pacientes presentaban ambos diagnósticos.

Los resultados mostraron que los factores que más se relacionaban con un trastorno de deglución severo eran: sexo masculino, limpieza oral incompleta, manejo anormal de la saliva, movilidad palatina anormal, saliva moviéndose libremente en glotis, disminución de la sensibilidad en la glotis, restos en seno piriforme y ahogo al comer.

Se propone un protocolo de evaluación de deglución para este tipo de pacientes acorde a nuestra experiencia como Servicio de Otorrinolaringología.

Palabras claves: Trastorno de deglución, factores de riesgo, nasofibroscopía.

\section{SUMMARY}

The deglution disorder is an increasing pathology among hospitalized patients. The study and handling of this condition has many alternatives according to the bibliography. We analyzed the inter-consults for deglution evaluations sent to our service between January 1st. and December 31st., 2004. A protocol was built with the data and the examination made and the patients were classified according to the severity of the disorder, thus entering into an algorithm designed to continue its therapy and follow-up.

\footnotetext{
${ }_{1}^{1}$ Medico Servicio Otorrinolaringología Hospital Cínico Universidad de Chile.

${ }^{2}$ Médicos Grujanos Universidad de Chile.
} 
This work could prove the high incidence of the deglution disorder in hospitalized patients; corresponding to $20 \%$ of the total inter-consults of the Service. The two most frequent causes for this disorder were: vascular accident in 56\% and extended intubation in $58 \%$ of the cases; some patients presented both diagnoses.

The results showed that the factors mostly related to a severe deglution disorder were: masculine sex, incomplete oral cleanness, abnormal handling of the saliva, abnormal palatine mobility, saliva moving freely in glottis, decrease of sensitivity in glottis, remains in pyriform apertures and suffocation when eating.

A deglution evaluation protocol is proposed for this type of patients, in accordance with our experience as Otorhinolaryngological Service.

Key words: Deglution disorder, risk factors, nasofibroscopy.

\section{INTRODUCCIÓN}

日 trastorno de deglución es un desorden en el transporte del bolo alimenticio desde la boca al estómago ${ }^{1}$. Se puede presentar en el contexto de alteraciones congénitas, traumas, lesiones cerebro vasculares o neurales, intubación prolongada, infecciones y otras 2 .

Parael estudio del trastorno de la deglución se debe partir con la anamnesis, evaluando la presencia, dura ción y tipo de disfagia, para luego realizar un examen físico completo con hincapié en el examen otorrinolaringológico, pulmonar y neurológico ${ }^{3}$. Esta evaluación tiende a ser subjetiva e incompleta por la ausenciade protocolos establecidos, el desconocimiento delos factores deriesgo relevantes en laaparición del trastorno de deglución o la sobrevaloración de los hallazgos nasofibroscópicos por sobre la anamnesis.

Se plantea el uso de exámenes complementarios como: esofagograma con bario, esofagograma contrastado con aire, cintigrama de bolo, ultrasonografía, videofluoroscopía y nasofibroscopía 4 .

La videofluoroscopía es el gold standard en el estudio de esta enfermedad. Se realiza con un fluoroscopio, 2 radiólogos, 1 otorrinolaringólogo 0 un fonoaudiólogo. Las ventajas de este examen son la facilidad de interpretación de todas las fases de la deglución; la evaluación de los movimientos compensatorios, y del tamaño y consistencia del bolo que el paciente maneja Sus desventajas son: que es un examen complejo de ejecutar, donde el paciente recibe radiación, debe colaborar y además es un examen prolongado en el tiempo ${ }^{5}$.

La nasofibroscopía requiere sólo de un naso- fibroscopio y un otorrinolaringólogo entrenado. Su ventaja es que evalúa el tamaño y consistencia del bolo que puede manejar el paciente. Es un examen barato, corto y no requiere radiación. La mayor ventaja es que se puede realizar incluso en pacientes postrados. Sin embargo, la nasofibroscopía no observa directamente la aspiración, no evalúa el esfínter cricofaríngeo ni la fase oral 6 .

La intubación prolongada produce trastorno de la deglución por daño delamucosalaríngea, quealteralos mecanorreceptores y quimiorreceptores; también produce hipotonía muscular por desuso y efecto narcótico. Se ha observado, que el trastorno de deglución se presenta más frecuentemente, cuando el paciente ha presentado en algún momento de su evaluación un glasgow menor a 14 puntos, si es un paciente mayor a 55 años, o que lleva menos de 96 horas extubado 7 . La bibliografía menciona que en pacientes con intubación prolongada se espera entre un $10 \%$ a $30 \%$ de aspira ción silente y entre un 5 a $20 \%$ de trastorno de la deglución, el cual estaráresuelto en un $63 \%$ delos casos sin mediar intervención algunaal mes de extubado8.

Los pacientes con accidente vascular encefálico presentan trastorno de la deglución por alteración neurológica ehipertensión endocraneana Son factores de riesgo para presentar mortalidad secundaria a trastornos de la deglución en pacientes con accidentes vasculares encefálicos la presencia de déficit de conciencia, disfagia, incontinencia urinaria, presencia de temperatura mayor a $36^{\circ} \mathrm{C}$, hiperglicemia sin diabetes. La disfagia será más frecuente en aquellos pacientes varones mayores de 70 años, asimetría del paladar, limpieza oral incompleta, respuestade tos disminuiday 
gárgaras $^{9}$. En los pacientes con accidentes vasculares encefálicos, según la bibliografía, la mejoría es parcial al mes del tratamiento en un $50 \%$ de los pacientes y a los 6 meses el $50 \%$ de los pacientes pueden estar sanos. Según estos datos se espera que un alto porcentaje de pacientes con accidente vascular encefá lico requieran gastrostomía ${ }^{10}$.

\section{OBJETIVOS}

Los objetivos de nuestro estudio fueron:

1. Conocer la epidemiología de los pacientes enviados para estudio de deglución.

2. Reconocer en nuestra población estudiada la presencia de factores de riesgo para trastorno de la deglución.

3. Proponer un protocolo de evaluación del trastorno de deglución que fuera realizable en nuestro medio.

\section{METODOLOGÍA}

Se revisaron todas las interconsultas de evaluación de deglución dirigidas al Servicio de Otorrinolaringología del Hospital Cínico de la Universidad de Chile desde el 1 de enero al 31 de diciembre de 2004. Todas las evaluaciones se realizaron por los investigadores médicos y se ingresaron a un protocolo que incluía dirigidamente la evaluación de factores de riesgo para aspiración.

En una primera evaluación se realizó una anamnesis completa, examen otorrinolaringológico y nasofibroscopía convencional y funcional, la cual consistíaen evaluar la deglución a la altura del paladar blando con ayuda de líquido teñido con azul de metileno y la sensibilidad laríngea mediante el contacto de la supra glotis con el nasofibroscopio. Con esta información se completó el protocolo presentado en el Anexo 1.

La conducta a seguir se efectuó de acuerdo a un diagrama preestablecido (Figura 1). En cada reevaluación se actualizaba la anamnesis y se volvía

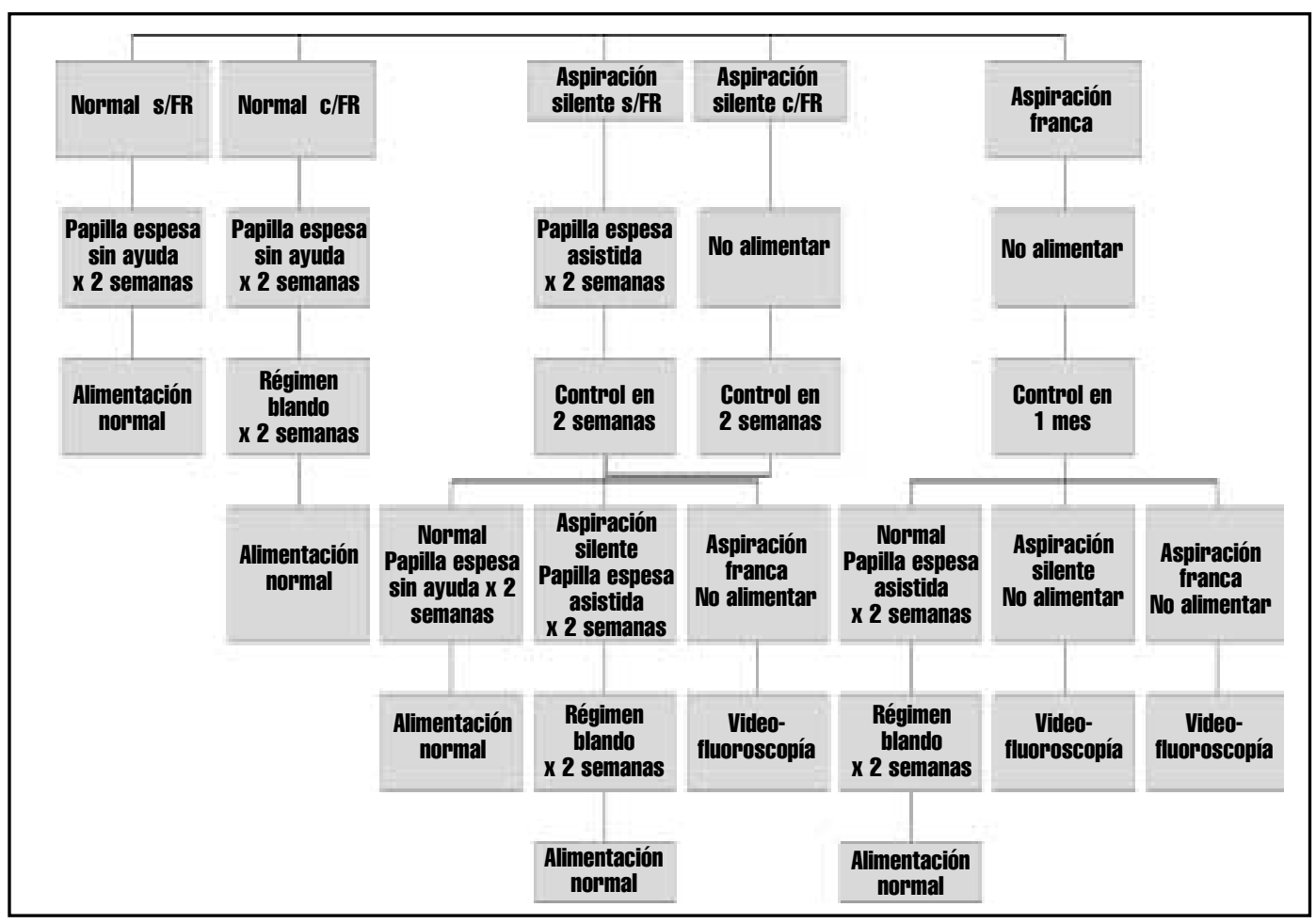

Figura 1. Tratamiento a seguir según la evaluación del proceso de deglución. $\mathrm{R}$ = Factor riesgo. 
a repetir el examen físico, nasofibroscopía general y funcional, colocando estos datos en el protocolo.

Al terminar nuestro periodo de investigación se contactó a los pacientes para terminar la información que requería el protocolo; preguntando la condición actual, si se alimentaban por boca y si presentaron neumonías durante el tiempo de seguimiento.

Para el estudio estadístico se caracterizó a la población según: sexo, edad y diagnóstico de ingreso más frecuente.

日 estudio estadístico se realizó con test de tstudent con un intervalo de confianza de $p>0,05$.

\section{RESULTADOS}

En el año 2004 seevaluaron en el Servicio de Corrinolaringologíaun total de 438 interconsultas de las cuales $20 \%$ correspondió a algún trastorno de la deglución.

Para la evaluación de este trabajo se consideraron 65 pacientes de los cuales se pudo obtener todos los datos que incluía el protocolo.

日 54\% correspondió al sexo masculino y el $46 \%$ al femenino.

La edad promedio fue de 62,2 años; y el rango de edad comprendió entre los 29 a los 92 años.

La aplicación del protocolo completo no complicó ni alargó considerablemente la evaluación (aproximadamente $15 \mathrm{~min}$ ). La nasofriboscopía convencional y funcional se logró realizar en el $100 \%$ de los pacientes con $0 \%$ de complicaciones.

Tabla 1. Diagnóstico de ingreso más frecuente

\begin{tabular}{|lr|}
\hline Diagnóstico & Porcentaje \\
\hline Accidente vascular encefálico & 44,6 \\
Hipertensión arterial & 41,5 \\
Enfermedad neurológica (distinta del AVE) & 41,5 \\
Patología infecciosa & 35,3 \\
Cardiovascular & 30,7 \\
Diabetes & 23 \\
\hline
\end{tabular}

Los porcentajes no son sumatorios; ya que algunos pacientes presentaban más de un diagnóstico.
Los diagnósticos de ingreso más frecuentes se mencionan en la Tabla 1. Cabe destacar que el $56 \%$ de la población en estudio presentó accidente vascular encefálico dentro de sus antecedentes y el $58 \%$ de los pacientes tuvo intubación prolongada.

Los pacientes se distribuyeron en grupos según la gravedad del trastorno de deglución de acuerdo a la Tabla 2. Se explica en el Anexo 1 los conceptos que implica cada categoría.

Los factores de riesgo encontrados con mayor frecuencia en los pacientes con AVEy trastorno de la deglución fueron: sexo masculino y presentar limpieza oral incompleta (Figura 2), con una diferencia estadísticamente significativa.

日 factor de riesgo más importante observado en los pacientes que tuvieron intubación prolongada y trastorno de la deglución más severo fue haber sido extubado hacía menos de 96 horas al momento de la evaluación (Figura 3).

Los hallazgos en el examen físico que se asociaron más frecuentemente a pacientes con deglución severamente alterada fueron: manejo de la saliva anormal y movilidad palatina anormal (Figura 4). Estos signos presentaron diferencia estadística con respecto a los otros grupos.

Los hallazgos más frecuentemente encontrados en la nasofibroscopía general, que se asociaron a evaluación de la deglución alterada fueron: saliva retenida en senos piriformes y menor sensibilidad de la glotis al tacto (Fgura 5); la evaluación estadística mostró que existía una diferencia significativa.

Los signos que se observaron en la nasofibroscopía funcional en los pacientes con peor evaluación de la deglución fueron: restos de comida en el seno piriforme, ahogo al comer (Figura 6); esta diferencia fue estadísticamente significativa.

\section{Tabla 2. Distribución de la población} según evaluación de la deglución

\begin{tabular}{lr|}
\hline Normal sin factores de riesgo & $26 \%$ \\
Normal con factores de riesgo & $28 \%$ \\
Aspiración silente sin factores de riesgo & $6 \%$ \\
Aspiración silente con factores de riesgo & $17 \%$ \\
Aspiración franca & $23 \%$ \\
\hline
\end{tabular}




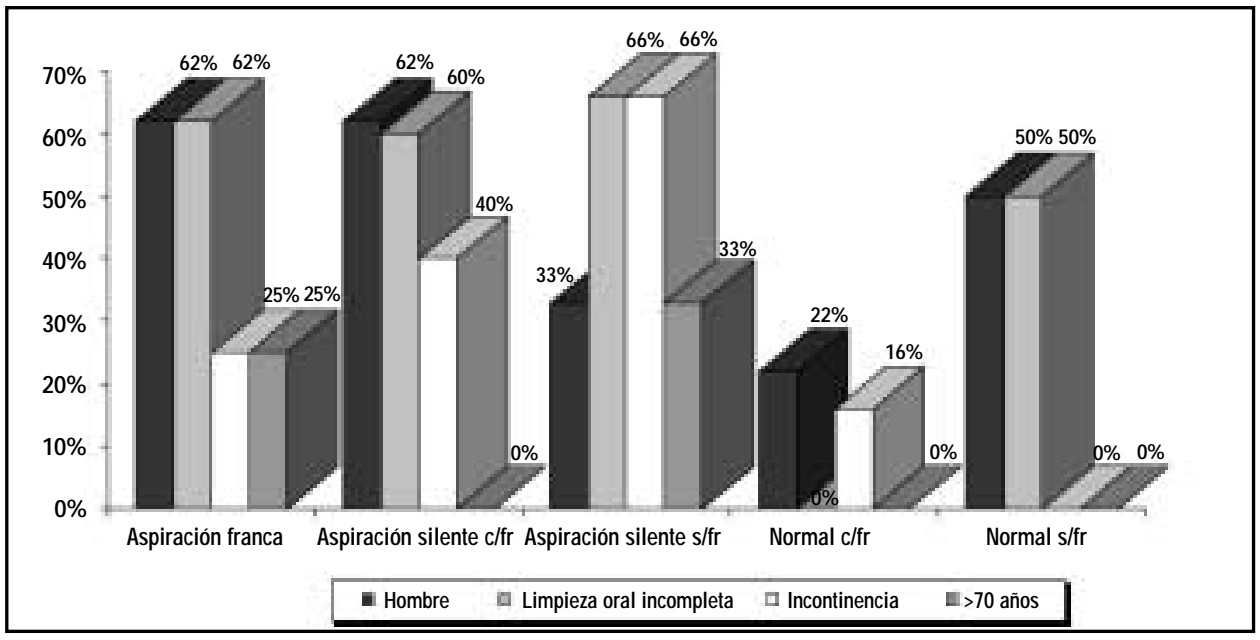

Figura 2. Factores de riesgo en pacientes con trastorno de deglución y accidente vascular encefálico.

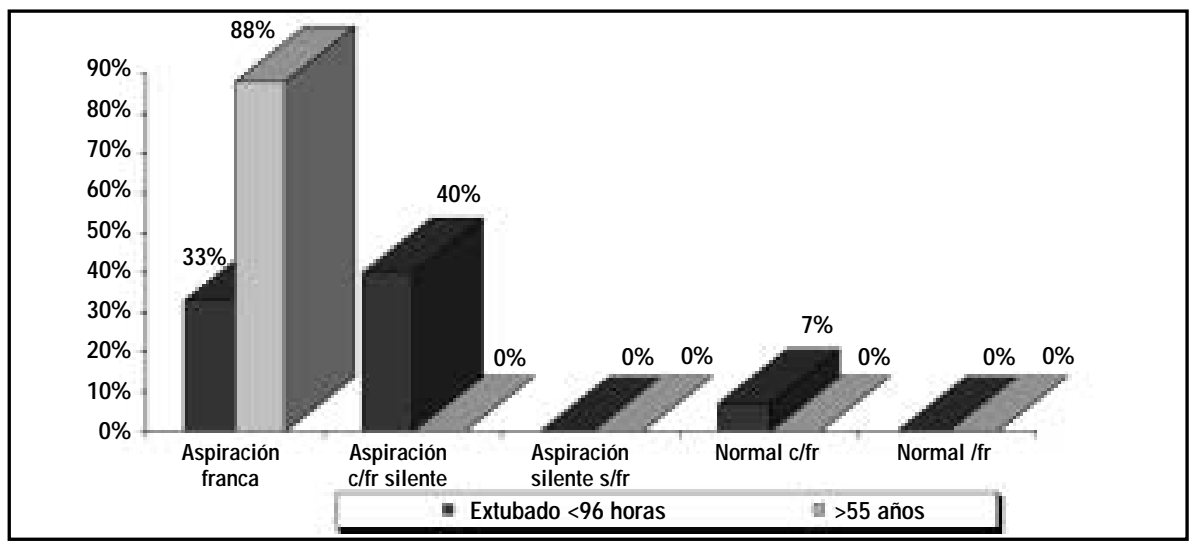

Figura 3. Factores de riesgo en pacientes con trastorno de deglución e intubación prolongada.

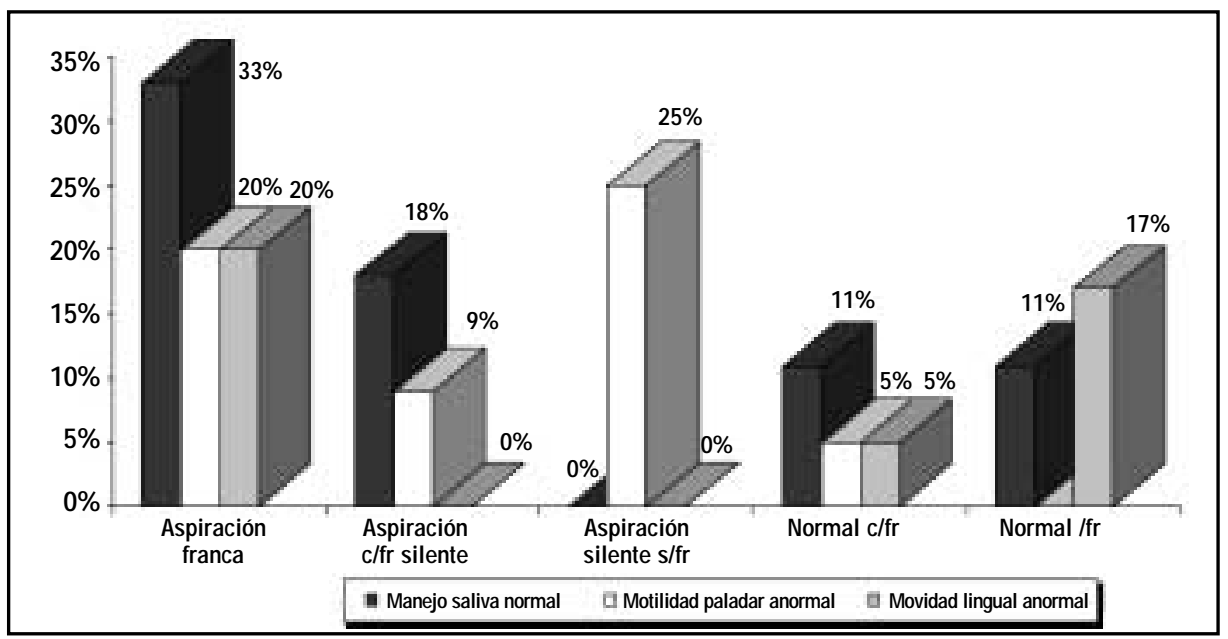

Figura 4. Hallazgos en el examen físico según la evaluación del trastorno de deglución. 
Anexo 1

Protocolo evaluación deglución

Fecha interconsulta:

Nombre:Ficha:

Edad: Diagnósticos:

\begin{tabular}{|c|c|c|c|c|c|c|}
\hline \multicolumn{7}{|c|}{ Antecedentes clínicos (si o no, indicar $N^{\circ}$ de días) } \\
\hline Antecedente & \multicolumn{2}{|c|}{ Primer control } & \multicolumn{2}{|c|}{ Segundo control } & \multicolumn{2}{|c|}{ Tercer control } \\
\hline AVE & $\mathrm{Si}$ & No & $\mathrm{Si}$ & No & $\mathrm{Si}$ & No \\
\hline Intubado & $\mathrm{Si}$ & No & $\mathrm{Si}$ & No & $\mathrm{Si}$ & No \\
\hline Tos & $\mathrm{Si}$ & No & $\mathrm{Si}$ & NO & $\mathrm{Si}$ & No \\
\hline Ahogo & $\mathrm{Si}$ & No & $\mathrm{Si}$ & No & $\mathrm{Si}$ & No \\
\hline Desaturación & $\mathrm{Si}$ & No & $\mathrm{Si}$ & No & $\mathrm{Si}$ & No \\
\hline \multicolumn{7}{|c|}{ Factores de riesgo en AVE: Solo llenar si presentó AVE } \\
\hline & \multicolumn{2}{|c|}{ Primer control } & \multicolumn{2}{|c|}{ Segundo control } & \multicolumn{2}{|c|}{ Tercer control } \\
\hline Conciente & $\mathrm{Si}$ & No & & No & $\mathrm{Si}$ & № \\
\hline Incontinencia urinaria & $\mathrm{Si}$ & No & $\mathrm{Si}$ & No & $\mathrm{Si}$ & No \\
\hline $\mathrm{T}^{\circ}>36,5$ & $\mathrm{Si}$ & No & $\mathrm{Si}$ & No & $\mathrm{Si}$ & No \\
\hline$>70$ años & $\mathrm{Si}$ & No & $\mathrm{Si}$ & No & $\mathrm{Si}$ & No \\
\hline Hiperglicemia sin DB & $\mathrm{Si}$ & No & $\mathrm{Si}$ & No & $\mathrm{Si}$ & No \\
\hline Hombre & $\mathrm{Si}$ & No & $\mathrm{Si}$ & No & $\mathrm{Si}$ & No \\
\hline Limpieza oral incompleta & $\mathrm{Si}$ & No & $\mathrm{Si}$ & No & $\mathrm{Si}$ & No \\
\hline Asimetría del paladar & $\mathrm{Si}$ & No & $\mathrm{Si}$ & No & $\mathrm{Si}$ & No \\
\hline Reflejo de tos & $\mathrm{Si}$ & No & $\mathrm{Si}$ & No & $\mathrm{Si}$ & No \\
\hline \multicolumn{7}{|c|}{ Factores de riesgo post-intubación: Solo Ilena si estuvo intubado } \\
\hline & \multicolumn{2}{|c|}{ Primer control } & \multicolumn{2}{|c|}{ Segundo control } & \multicolumn{2}{|c|}{ Tercer control } \\
\hline Intubación $>48$ horas & $\mathrm{Si}$ & No & $\mathrm{Si}$ & No & $\mathrm{Si}$ & No \\
\hline Extubación >96 horas & $\mathrm{Si}$ & No & $\mathrm{Si}$ & No & $\mathrm{Si}$ & No \\
\hline Gasgow $<14$ & $\mathrm{Si}$ & No & $\mathrm{Si}$ & No & $\mathrm{Si}$ & No \\
\hline$>55$ años & $\mathrm{Si}$ & No & $\mathrm{Si}$ & No & $\mathrm{Si}$ & No \\
\hline \multicolumn{7}{|c|}{ Examen físico (normal o anormal, indicar \% de saturación) } \\
\hline & \multicolumn{2}{|c|}{ Primer control } & \multicolumn{2}{|c|}{ Segundo control } & \multicolumn{2}{|c|}{ Tercer control } \\
\hline Saturación & $<90 \%$ & $>90 \%$ & $<90 \%$ & $>90 \%$ & $<90 \%$ & $>90 \%$ \\
\hline Tono paladar & Normal & Anormal & Normal & Anormal & Normal & Anormal \\
\hline Motilidad palatina & Normal & Anormal & Normal & Anormal & Normal & Anormal \\
\hline Movimientos de lengua & Normal & Anormal & Normal & Anormal & Normal & Anormal \\
\hline Manejo bucal de saliva & Normal & Anormal & Normal & Anormal & Normal & Anormal \\
\hline \multicolumn{7}{|l|}{ Nasofibroscopía convencional } \\
\hline & \multicolumn{2}{|c|}{ Primer control } & \multicolumn{2}{|c|}{ Segundo control } & \multicolumn{2}{|c|}{ Tercer control } \\
\hline Saliva retenida en laringe & $\mathrm{Si}$ & No & $\mathrm{Si}$ & No & & No \\
\hline Motilidad de las CV & Normal & Anormal & Normal & Anormal & Normal & Anormal \\
\hline Sensibilidad al contactar glotis & Normal & Anormal & Normal & Anormal & Normal & Anormal \\
\hline
\end{tabular}

Nasofibroscopia funcional: Se evalúa al dar de tomar agua $5 \mathrm{cc} ; 3 \mathrm{cc}$, y se observa sobre la epiglotis

Tos al comer

Ahogo al comer

Expectoración al comer

Desaturación al comer

Restos en seno piriforme después de comer

\section{Primer control}

$\mathrm{Si} \quad$ No

$\mathrm{Si} \quad$ No

$\mathrm{Si} \quad$ No

$\mathrm{Si} \quad$ No

$\begin{array}{lc}\text { Segundo Control } \\ \text { Si } & \text { No } \\ \mathrm{Si} & \text { No } \\ \mathrm{Si} & \text { No } \\ \mathrm{Si} & \text { No } \\ \mathrm{Si} & \text { No }\end{array}$

Tercer Control

$\mathrm{Si} \quad$ No

$\mathrm{Si} \quad$ No

$\mathrm{Si} \quad$ No

$\mathrm{Si} \quad \mathrm{No}$

Si No 
Resultado ( $\mathrm{R}=$ factores de riesgo; marcar el Dg del control y conducta)

Antecedente

Normal s/FR

Primer control

Segundo control

Tercer control

Normal c/ $\mathrm{RR}$

Aspiración silente s/ $/ \mathbb{R}$

Aspiración silente c/ R

Aspiración franca

Llamada telefónica

No teléfono:

$\mathrm{Si}$

No

Vivo

Come por boca

Ha tenido neumonías

Se define el resultado del examen como:

Normal: será aquel examen donde no hay clínica de aspiración (tos, cianosis, desaturación que se presente en el proceso de deglución) ni hay presencia en la nasofibroscopía de residuos de alimento en la supraglotis.

Aspiración silente: se presenta como aquel evento en que la deglución no se acompaña de síntomas o signos característicos de trastorno de la deglución pero en la nasofibroscopía existen residuos en la glotis que el paciente no es capaz de limpiar.

Aspiración franca: es aquella evaluación en la cual existirá clínica de trastornos de deglución y en la nasofibroscopía existirán residuos en la glotis que el paciente no es capaz de limpiar e incluso se verán estos residuos en la subglotis.

Sin factores de riesgo: esto implica aquel paciente que presenta menos de 2 factores de riesgo en la evaluación.

Con factores de riesgo: serán aquellos pacientes en cuya evaluación tienen 3 o más factores de riesgo.

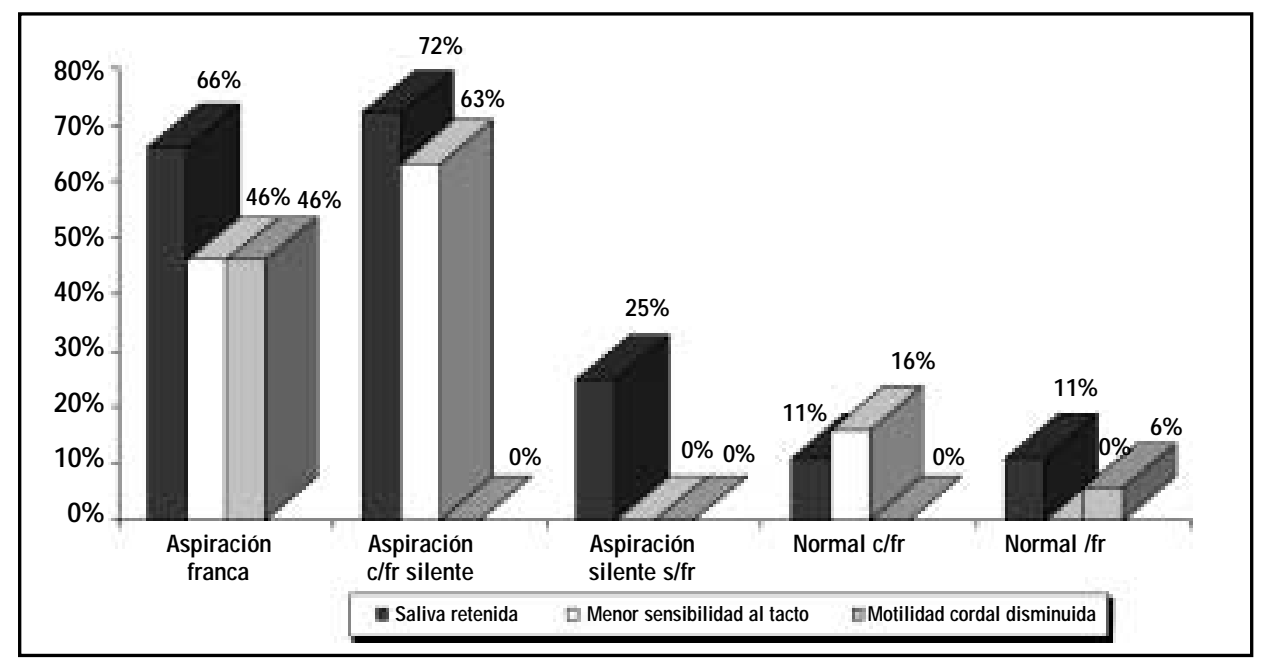

Figura 5. Hallazgos en la nasofibroscopía general según la evaluación del trastorno de deglución.

Después de un seguimiento promedio de 7 meses con un rango de 1 a 12 meses; se observó que la mortalidad y la morbilidad por neumonía y alimenta ción no oral fueron mayores en los grupos que, en la primera evaluación de la deglución, mostraron un estado más alterado (Figuras 7 y 8); el estudio estadístico mostró que esta diferencia era significativa

\section{DISCUSIÓN}

日 trastorno de deglución es una patología que ha ido aumentando su prevalencia a lo largo del tiempo, debido al incremento en la esperanza de vida mundial y a los avances médicos que han reducido la mortalidad de algunas patologías. Es 


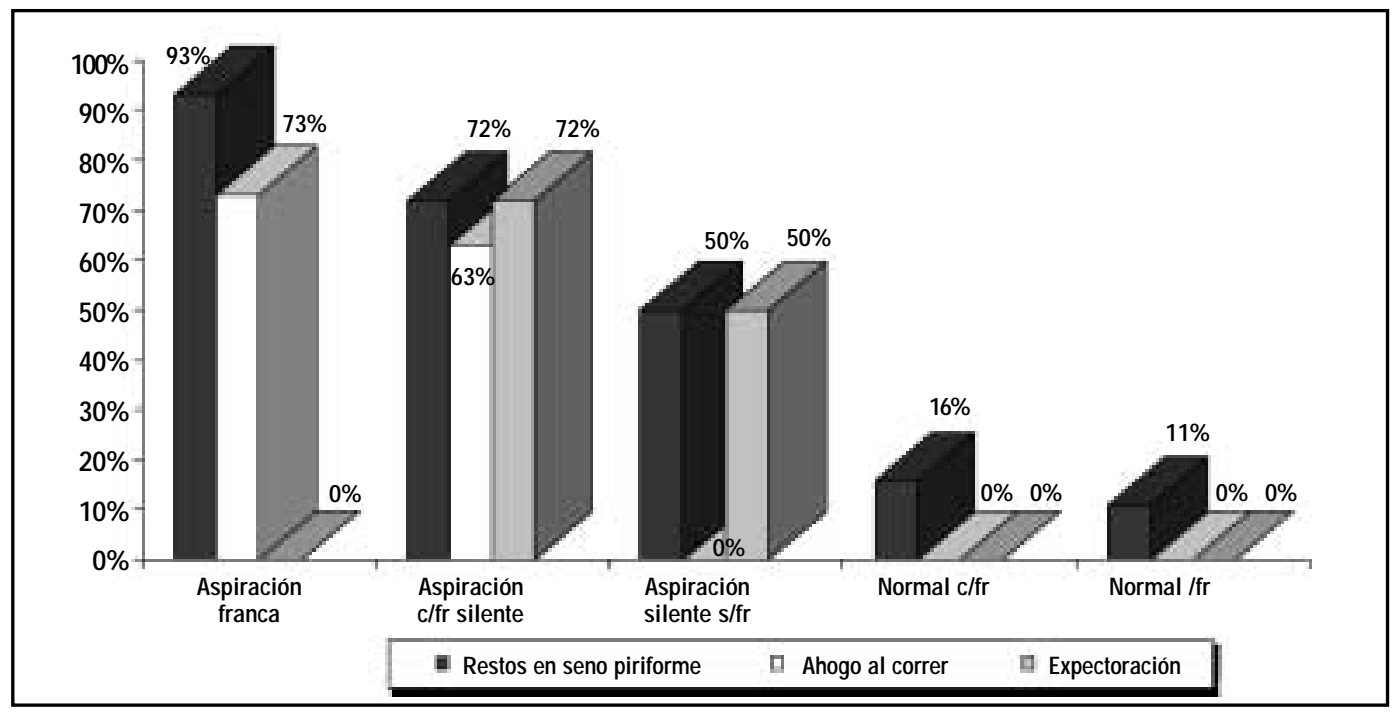

Figura 6. Hallazgos en la nasofibroscopía funcional según la evaluación del trastorno de deglución.

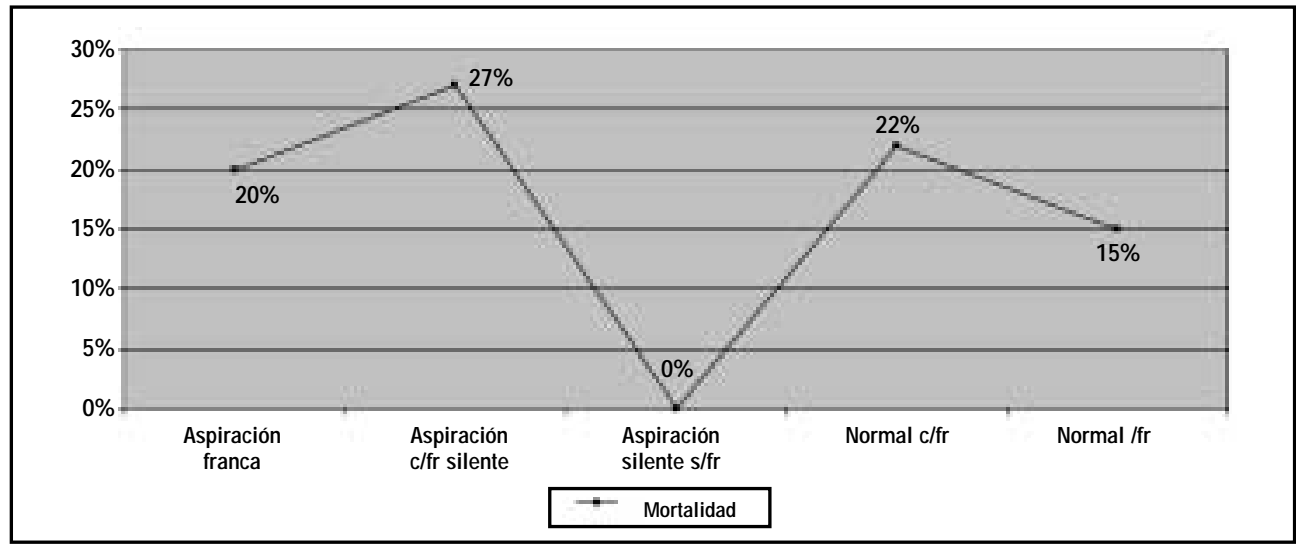

Figura 7. Mortalidad según la gravedad en el trastorno de deglución.

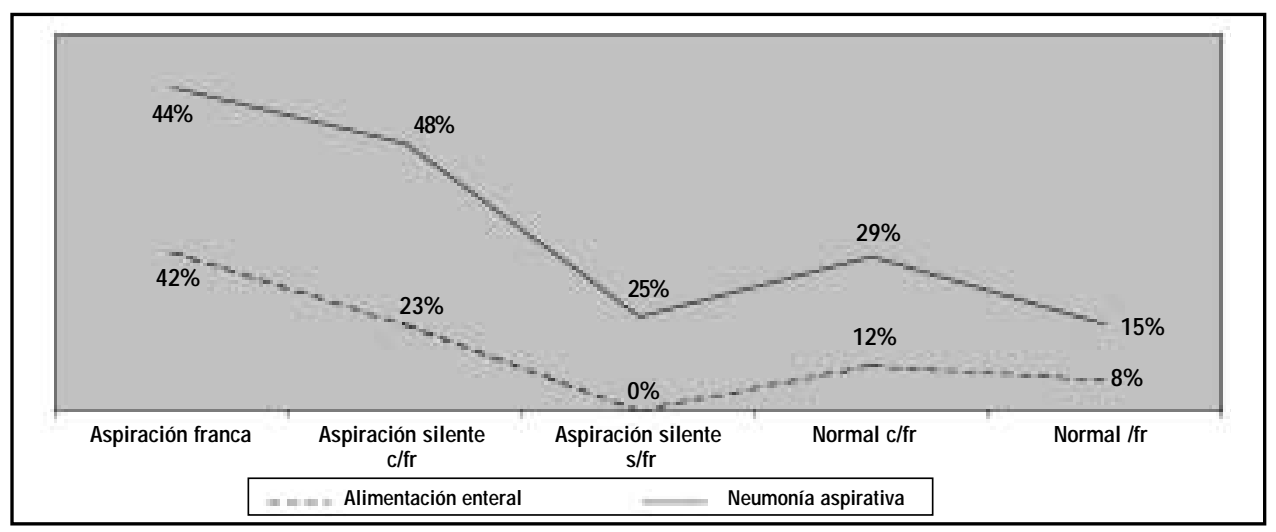

Figura 8. Morbilidad según la gravedad en el trastorno de deglución. 
por esto la importancia de su estudio y manejo adecuado para reducir la morbimortalidad que ésta acarrea.

Nuestro trabajo ha mostrado que la incidencia de esta patología es importante siendo la causal del $20 \%$ de las solicitudes de interconsultas a nuestro servicio.

Nuestra población en estudio tiene un promedio de edad de 62 años; leve predominancia masculina y con diagnóstico de ingreso en que predomina el accidente vascular encefálico; lo que es compatible con la bibliografía.

Nuestro grupo de trabajo insiste que la evaluación de la deglución debe ser integral; comenzando con la anamnesis; donde se observa que el sexo masculino y presentar limpieza oral incompleta ya nos deben hacer sospechar que un paciente con accidente vascular encefálico pueda desarrollar un severo trastorno en la deglución. Así mismo, los pacientes con intubación prolongada que han sido extubados hace menos de 96 horas; deben sugerir un trastorno de deglución.

En el examen físico se observó que aquellos pacientes que tenían manejo de la saliva y movilidad del paladar anormal presentaban trastornos severos de la deglución, todos ellos factores evaluables en el examen otorrinolaringológico general sin necesidad siquiera de exámenes más complejos.

Los otros factores de riesgo que se estudiaron en la anamnesis y examen físico no tenían relación directa con la severidad del trastorno; si bien es bueno destacar que la sumatoria de factores de riesgo también podría asociarse con un trastorno de la deglución más severo.

En la nasofibroscopía general tenemos los factores de riesgo más relevantes para la evaluación de la deglución y que tuvieron mayor asociación con trastornos severos de la deglución. Estos son: observar saliva moviéndose libremente en senos piriforme y glotis; el poder tocar la glotis con la punta del nasofibroscopio sin producir reacciones de defensa del individuo. Todo esto posicionaa la nasofibroscopía como una excelente herramienta de evaluación de la deglución.

La certeza diagnóstica del trastorno de la deglución se realizó con la nasofibroscopía funcional donde se daba a ingerir agua con azul de metileno y se observaba la presencia de aspiración franca o ahogo.

\section{CONCLUSIÓN}

Nuestro trabajo confirma que la evaluación de la deglución, como cualquier otra patología, es un proceso que empieza en la anamnesis; clave para la morbimortalidad del paciente y que, por lo tanto, debe ser completo y exhaustivo. No podemos tomar la decisión de realimentación oral de estos pacientes sólo considerando la nasofibroscopía y desconociendo los otros factores de riesgo de la historia o del examen general. Esto no significa que se convierta en un examen largo y tedioso ya que teniendo claro los factores más relevantes en cada etapa de la evaluación, puede convertirse en un procedimiento corto y efectivo.

Dejamos en conocimiento de los otorrinolaringólogos una herramienta para evaluar la de glución diseñada por nosotros y para nuestra realidad. Sin embargo, creemos que puede ser de utilidad en el camino de aunar criterios para este tipo de evaluación.

En cuanto al seguimiento con nuestro algoritmo de tratamiento, solo alcanzó a un 20\%; por lo que no fue posible analizarlo estadísticamente. Esto se debió a que nuestros pacientes nos son derivados para evaluación postoperatoria en forma rutinaria; ya que el tratamiento a seguir muchas veces es decidido por su médico de cabecera sin consultarnos. Se mejorará este aspecto para que en un futuro podamos validar también este algoritmo terapéutico.

\section{BIBLIOGRAFÍA}

1. LoGeMANN J. Evaluation and treatment of swallowing disorders. San Diego College-Hill Press, 1983.

2. KIM OH, HSUJJ, WILIAMS DEET AL. A prospective psychological evaluation of patients with dyspha gia of various etiologies. Dysphagia 1996; 11: 34. 
3. GARY L SCHECHIIR, MD. Systemic causes of disphagia in adults. Otolaryngologic Cinics of North America 1998; 31: 525-34.

4. ROBERT W BASTIAN, MD. Contemporary diagnosis of the disphagic patient. Aolaryngologic Cinics of North America 1998; 31: 489-506.

5. MeNDESOHN RS, Mc CONNE RMS. Function in the phryngoesophageal segment. Laryngoscope 1987, 97: 483-9.

6. LIM SH, LIE PK ET AL. Accuracy of bedside clinical methods compared with fiberoptic endoscopic examination of swallowing (זES) in detemining the risk of aspiration in acute stroke patients. Dysphagia 2001; 16 (1): 1-6.

7. BarQuist EMD, Brown M, MSN, et Al. Postextubation fiberoptic endoscopic evaluation of swallowing after prolonged endotracheal intubation: A randomized, prospective trial. Crit Care Med 2001; 29: 1710-13.

8. Augmian MS, Nirmul BG et al. Routine fiberoptic endoscopic evaluation of swallowing following prolonged intubation: Implication for management. Arch Surg 2001; 136 (4): 434-7.

9. WANG Y, LIM LL ET AL. A prognostic index for 30 day mortality after stroke. J Cin Epidemiol 2001; 54 (8): 766-73.

10. EBLING D, MD, CARRAU R, MD. Detection, evalua tion and management of aspiration in rehabilita tion hospitals: Role of the Otolaryngologist-Head and Neck Surgeon. The Journal of Otolaryngology 2001; 30 (4): 235-41.

Dirección: Dra. Patricia Arteaga

Servicio Otorrinolaringología, Hospital Cínico Universidad de Chile.

Santos Dumont 999. Tel: 9788153.

Email: patty_arteaga@hotmail.com 